\title{
Nano/bio interface:impact on drug delivery applications
}

\author{
Subhra Mohapatra • Srinivas Nagaraj • \\ Shyam S. Mohapatra
}

Published online: 30 April 2013

(C) Controlled Release Society 2013

With the advent of the rapidly growing field of nanotechnology that has ushered new opportunities to develop multifunctional biomedical nanoparticles, nanoscientists are learning about the extra- and intracellular interactions with these nanoparticles. This special issue of Drug Delivery and Translational Research is dedicated to publishing selected deliberations relating to drug delivery in the first NanoBio Collaborative International Conference 2012 (NBCIC 2012) that was organized by the University of South Florida Nanomedicine Research Center and was held in March 22-24, 2012.

The goal of this conference was to promote closer research ties and networking opportunities within the nanoscience community of the State of Florida and to foster and encourage collaborative research efforts among national and international centers of excellence in nanotechnology. NBCIC 2012 brought together engineers, chemists, physicists, biologists, and clinicians from across the globe to discuss recent advances, opportunities, and barriers in nanoscience and nanotechnology and its applications to diagnosing and treating disease. The conference was comprised of several symposia, including nanobioengineering applications, cell technologies and regenerative medicine, theranostics, nanodrug delivery, and translational nanomedicine. This special issue presents four state-of-the-art reviews and four research articles

S. Mohapatra $(\bowtie)$

Department of Molecular Medicine, and USF Nanomedicine Research Center, Morsani College of Medicine, University of South Florida, Tampa, FL, USA

e-mail: smohapa2@health.usf.edu

\section{S. Nagaraj $\cdot$ S. S. Mohapatra}

Division of Translational Medicine, Department of Internal Medicine, and USF Nanomedicine Research Center, Morsani College of Medicine, University of South Florida, Tampa, FL, USA

$$
\begin{aligned}
& \text { S. Nagaraj } \\
& \text { e-mail: snagaraj@health.usf.edu } \\
& \text { S. S. Mohapatra } \\
& \text { e-mail: smohapat@health.usf.edu }
\end{aligned}
$$

highlighting the application of nanotechnology to drug delivery that were discussed in the conference.

Sir Harold Kroto, Nobel Laureate in Chemistry (1996), was a Keynote Speaker discussing his vision of nanoscience and nanotechnology in the twenty-first century and has written a preface for this issue. He provided examples of technology use in the future from medicine and molecular electronics to civil engineering. Expanding on medical applications, he stated "the drug industry has focused on the production of relatively simple molecules... these molecules are now highlighting incredibly exciting new directions for molecular biological research and challenging new perspectives for pharmaceutical research and development".

The last decade has seen progress in development of biomaterials for tissue engineering applications; however, self-assembling peptide scaffolds especially for delivery of drugs to injured tissues or organs have been poorly investigated. The first review by Veronica et al. reviews the application of self-assembling peptide scaffolds as an innovative platform for drug and cell delivery to restore cardiac tissue after myocardial infarction.

Targeted drug delivery has been the holy grail of cancer therapy. The review article by Yin et al. discusses cyclodextrinbased supramolecules, polymers, and nanoparticles bearing targeting moieties such as folates, estrogens, carbohydrates, and peptides as a promising drug delivery system.

While effective delivery of therapeutics to the central nervous system (CNS) remains a formidable challenge, the article by Shah et al. reviews the major barriers to CNS delivery and nanotechnology-based solutions for effective drug delivery to the CNS. The article discusses different forms of nanoparticlebased drug delivery systems with emphasis on different modes of delivery to the brain and their safety issues.

Last but not least, theranostic nanoparticles that combine both therapeutic and imaging modalities has the promise to revolutionalize diagnosis, treatment, and post-therapy monitoring. The review of Howell et al. summarizes recent developments in theranostic platforms, their advantages and disadvantages in the diagnosis and treatment of various 
lung-related diseases and their potential application to chronic lung diseases.

Beyond these reviews, the compendium also contains research articles on drug delivery systems and mechanisms. With an intent to enhance drug accumulation in target cells, the original research article by Kumar et al. reports a singlestep synthesis of a "hybrid lipid-PLGA based PEGylated nanoparticles" platform without the necessity of covalently linking the PLGA to PEG for encapsulating hydrophobic drugs. This robust platform was able to increase intracellular drug loading capacity by 22 times, thus enhancing the efficiency of drug delivery and imaging of cancer cells.

The research article by Bi et al. investigates the protective and anti-inflammatory effects of nanoparticles loaded with quercetin (Q-NPs) prepared with binary mixtures of cetyl alcohol and Gelucire. Both in vitro and in vivo studies suggest that Q-NPs possess intrinsic protective properties against LPS-induced inflammation.

The original research article by Mdzinarishvili et al. reports the feasibility and advantage of PLGA-PEG nanoparticles to deliver thyroid hormones for ischemic brain stroke, using middle cerebral artery occlusion.

The communication by Downing et al. establishes the mechanism of the protective effects of cerium oxide nanoparticles. Their results suggest that one of the protective effects of $\mathrm{CeO} 2 \mathrm{NPs}$ against reactive nitrogen species is due to reduction of peroxynitrite or its reactive breakdown products.

Acknowledgments We acknowledge the funding from the USF Conference Support Grant and the support from the Office of Research, Morsani College of Medicine and College of Pharmacy. 